\title{
Characterizing the dynamic range of a hyperspectral camera
}

\author{
Skauli, Torbjørn
}

Torbjørn Skauli, "Characterizing the dynamic range of a hyperspectral camera," Proc. SPIE 11727, Algorithms, Technologies, and Applications for Multispectral and Hyperspectral Imaging XXVII, 117270D (12 April 2021); doi: 10.1117/12.2588305

SPIE. Event: SPIE Defense + Commercial Sensing, 2021, Online Only 


\title{
Characterizing the dynamic range of a hyperspectral camera
}

\author{
Torbjørn Skauli*ab \\ ${ }^{a}$ University of Oslo, Dept. of Technology Systems, Gunnar Randers vei 19, 2007 Kjeller, Norway; \\ ${ }^{b}$ Norwegian Defence Research Establishment (FFI), Instituttveien 20, 2007 Kjeller, Norway
}

\begin{abstract}
The dynamic range of a hyperspectral camera can have a strong impact on the quality and integrity of the collected spectra. For the image sensor chip alone, the dynamic range is the ratio between the saturation level and the noise floor, set by full well capacity and readout noise respectively. In a complete hyperspectral camera, the raw signal level varies with the wavelength-dependent detector quantum efficiency, optics transmission, and illumination, as well as with the optical bandwidth. In practice, different parts of the spectrum will tend to have the highest or lowest signal level, and will most easily reach the saturation level or noise floor, respectively. It is shown that to define a dynamic range for a hyperspectral camera in the reflective spectral domain, the wavelength dependence of the camera light collection efficiency must be taken into account. A more application-oriented dynamic range can be defined by additionally assuming a shape of the illumination spectrum representative of a particular application.
\end{abstract}

Keywords: Imaging spectroscopy, Multispectral imaging, Saturation, Well capacity, Readout noise

\section{INTRODUCTION}

When recording hyperspectral images, an issue that is often encountered is how to set the integration time. If it is set too short, the signal to noise ratio will be unnecessarily low. And if the integration time is too long, some parts of the image cube will contain invalid data due to saturation. Often, the scene (or object) viewed by a hyperspectral camera exhibits large variations in the light level arriving in a given band and pixel, making it difficult to find an optimal integration time. In a remote sensing scene, for example, the light level in shadowed areas may be lower than in sunlit areas by orders of magnitude, and may vary over time. Also, the reflectance of scene materials may vary by a large factor across the spectral range of the camera. Not least, the illumination source in a given application will normally have a significant variation of light level with wavelength. It is therefore desirable for a hyperspectral camera to have a wide dynamic range, in order to accommodate signal variations in the scene.

The dynamic range is normally taken to be the ratio between the highest and lowest signal level that can be measured. For a regular camera, as well as for the image sensor inside the hyperspectral camera, this would be the ratio between the saturation level and the noise floor. In hyperspectral imaging, however, it is common to encounter situations where a pixel spectrum contains only a few bands in saturation, or a part of the spectral range is at the noise floor. In remote sensing, it is desirable to be able to collect the best possible signal near the edges of atmospheric transmission bands while avoiding saturation in bands where daylight illumination is strong. Clearly, it is desirable to have a meaningful way of characterizing the dynamic range of a hyperspectral camera that takes these effects into account.

The IEEE P4001 working group ${ }^{1}$ is developing a standard for hyperspectral imaging where camera characteristics is the main focus. The group has decided that dynamic range will be among the characteristics included. This paper presents a definition of dynamic range which is being considered for inclusion in the standard. The treatment here considers hyperspectral imaging of reflected light, in keeping with the P4001 charter. It is shown that in order to define the dynamic range in a meaningful way, it is necessary to take into account the wavelength dependence of camera sensitivity, and also to consider the shape of the illumination spectrum.

\section{SIGNAL MODEL.}

Saturation and noise are effects related to the electronic signal chain of a camera. It is therefore of interest here to establish a simple model for the raw signal in a hyperspectral camera. In practically all cases, the analog raw signal is a

*torbjorn.skauli@its.uio.no

Algorithms, Technologies, and Applications for Multispectral and Hyperspectral Imaging XXVII,

edited by Miguel Velez-Reyes, David W. Messinger, Proc. of SPIE Vol. 11727, 117270D

(C) 2021 SPIE · CCC code: 0277-786X/21/\$21 - doi: 10.1117/12.2588305 
"charge packet" of electrons excited by incoming photons, known as photoelectrons. Consider the signal in band $j$ of a pixel in a hyperspectral camera. Let the incoming signal be represented as a photon radiance $L_{\lambda, p h}(\lambda)$ with dimension photons/(area $\times$ solid anglexwavelength). To simplify the discussion here, it is assumed that the radiance has at most a linear spectral variation within the bandwidth $\Delta \lambda_{j}$ and at most a linear temporal variation within the integration time $t_{\text {int }}$ so that the input photon radiance can be represented by its value $L_{\lambda, p h}\left(\lambda_{j}\right)$ at the band center wavelength $\lambda_{j}$. The photoelectron count can then be written

$$
N_{e, j}=A_{\text {det }} \Omega_{\text {det }} T_{\text {opt }}\left(\lambda_{j}\right) \eta_{\text {det }}\left(\lambda_{j}\right) t_{\text {int }} \Delta \lambda_{j} L_{\lambda, p h}\left(\lambda_{j}\right) .
$$

Here, $A_{d e t}$ is the area of the corresponding detector element on the image sensor of the camera, $\Omega_{\text {det }}$ is the solid angle of the focused light cone incident on the detector, $T_{\text {opt }}\left(\lambda_{j}\right)$ is the transmission through the optics and $\eta_{\text {det }}\left(\lambda_{j}\right)$ is the quantum efficiency of the detector. The integration time $t_{\text {int }}$ is normally a user settable parameter. The bandwidth $\Delta \lambda_{j}$ can be chosen to meet application requirements, although for most hyperspectral camera technologies the choice must be made during design.

The product $A_{\text {det }} \Omega_{\text {det }}$ represents the throughput, or étendue, of the camera, while the product $T_{\text {opt }}(\lambda) \eta_{\text {det }}(\lambda)$ represents wavelength-dependent losses (assumed to vary slowly with wavelength). These four factors only act on the signal by their product. The notation can then be simplified by using the effective throughput $A^{*}$ with a slightly modified definition from that in [2]:

$$
A^{*}(\lambda)=A_{d e t} \Omega_{\text {det }} T_{o p t}(\lambda) \eta_{\text {det }}(\lambda) .
$$

The quantity $A^{*}(\lambda)$ is a well-defined function of wavelength, independent of bandwidth, and thus a compact way to specify wavelength-dependent light collection for any camera. (The definition in [2] could then be denoted $A_{\max }^{*}$.) The signal model can now be written somewhat more compactly as

$$
N_{e, j}=A^{*}\left(\lambda_{j}\right) t_{\text {int }} \Delta \lambda_{j} L_{\lambda, p h}\left(\lambda_{j}\right) .
$$

As an illustration, consider the left plot in Figure 1, which shows the quantum efficiency for a silicon-based image sensor and the diffraction efficiency of a grating. These components could be used in a hyperspectral camera for the spectral range shown, and would then tend to determine the wavelength dependence of the effective throughput, illustrated in the plot on the right. The qualitative features of this graph, where the effective throughput drops off at the ends of the spectral range, is typical of most hyperspectral cameras.
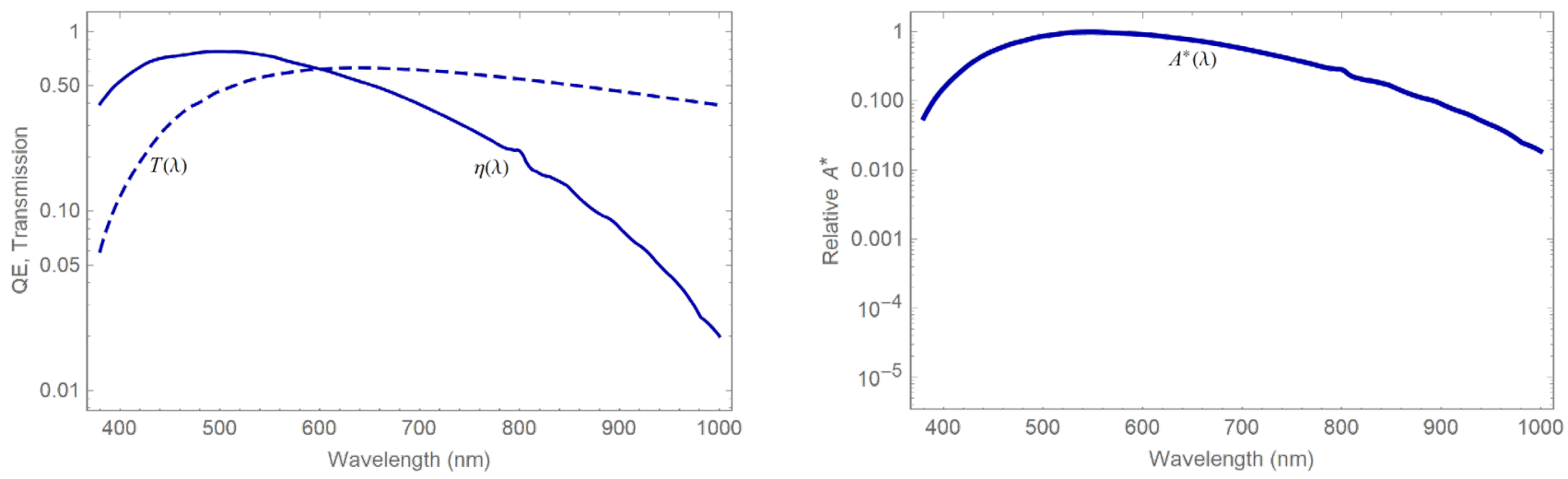

Figure 1. Illustration of the wavelength dependence of light collection in a hyperspectral camera. Left: The wavelength dependence of the quantum efficiency of a silicon-based image sensor and the diffraction efficiency of a grating in the visible and near-infrared spectral range. Right: Relative variation of the effective throughput for a hyperspectral camera where the graphs on the left determine the wavelength dependence.

Saturation can occur as a result of the analog-to-digital conversion reaching its maximum binary value, in which case saturation can be identified by the data value. However, saturation can also occur in the analog part of the signal chain, in 
which case the saturation behavior may be different and less predictable. Here, saturation is taken to be the photoelectron count $N_{\text {sat }}$ at which the raw signal no longer follows its nominal linear relation to the incoming light level. The saturation level for radiance in a given band can be found from (3) as

$$
L_{\lambda, p h, s a t, j}=\frac{N_{s a t}}{A^{*}\left(\lambda_{j}\right) t_{\mathrm{int}} \Delta \lambda_{j}} .
$$

Ideally, the noise in the signal from a camera is dominated by the Poisson statistics of photoelectron generation by the incoming photon flux. This "photon noise" in the photoelectron count has a standard deviation equal to the square root of the mean count. At low signal levels, the photon noise will be smaller than the noise floor set by residual noise from the analog signal chain of the camera, known as the "read noise". This electronic noise is normally expressed as an equivalent RMS fluctuation in the photoelectron count, here denoted $N_{\sigma r}$. Then, from (3) we get

$$
L_{\lambda, p h, \min , j}=\frac{N_{\sigma r}}{A^{*}\left(\lambda_{j}\right) t_{\mathrm{int}} \Delta \lambda_{j}}
$$

for the noise floor, expressed as an equivalent incoming radiance in a given band.

\section{DYNAMIC RANGE}

The dynamic range for a single band can be defined in the conventional way as

$$
D R_{\text {mono }}=\frac{N_{\text {sat }}}{N_{\sigma r}} .
$$

This will represent the dynamic range of the camera when the incoming light is monochromatic. The light level at which saturation occurs will vary inversely proportional to the effective throughput $A^{*}$ at the wavelength of the light signal. The spectral variation of the saturation level for the case shown in Figure 1 will therefore be as illustrated in Figure 2, assuming for simplicity that all bands have the same bandwidth. The wavelength dependence of the noise floor will have the same relative shape, as shown in the figure, at some low signal level. For the illustrative data in the figure, the ratio between saturation level and noise floor is about 5000. As illustrated in Figure 2 a), this will be the dynamic range for monochromatic light at any wavelength.

Monochromatic light is not a common use case for hyperspectral imaging. Consider instead a situation where the photon spectral radiance distribution is flat, with the same value at all wavelengths, illustrated in Figure $2 \mathrm{~b}$ ). When the light level is increased, saturation occurs first at the wavelength where the effective throughput is largest. When the light level is reduced, the noise floor is reached first at the wavelength with the lowest effective throughput. This suggests an alternate definition of dynamic range, which can be expressed as

$$
D R_{\text {cam }}=\frac{\frac{N_{\text {sat }}}{\max _{j}\left[A^{*}\left(\lambda_{j}\right) \Delta \lambda_{j}\right]}}{\frac{N_{\sigma r}}{\min _{j}\left[A^{*}\left(\lambda_{j}\right) \Delta \lambda_{j}\right]}}=\frac{N_{\text {sat }}}{N_{\sigma r}} \frac{\min _{j}\left[A^{*}\left(\lambda_{j}\right) \Delta \lambda_{j}\right]}{\max _{j}\left[A^{*}\left(\lambda_{j}\right) \Delta \lambda_{j}\right]} .
$$

The result is that the dynamic range in Figure 1 a) is reduced by a factor given by the relative variation of light collection over all bands. This could be taken as a natural characteristic of the camera dynamic range. Underlying the definition (7) is the assumption of a flat photon radiance spectrum. While this may be a reasonable way to characterize the camera, it is clearly not representative of a practical application.

In a practical case, the incoming light will tend to have a spectral distribution given by the illumination source, at least under a coarse "gray world" assumption where the average scene reflectance is taken to be spectrally flat. By assuming a particular shape $L_{\lambda, p h, \text { illum }}(\lambda)$ for the illuminant spectrum, it is possible to define a corresponding dynamic range as 


$$
D R_{\text {illum }}=\frac{\frac{N_{\text {sat }}}{\max _{j}\left[A^{*}\left(\lambda_{j}\right) \Delta \lambda_{j} L_{\lambda, p h, \text { illum }}\left(\lambda_{j}\right)\right]}}{N_{\sigma r}}=\frac{N_{\text {sat }}}{\min _{j}\left[A^{*}\left(\lambda_{j}\right) \Delta \lambda_{j} L_{\lambda, p h, \text { illum }}\left(\lambda_{j}\right)\right]} \frac{\min _{\sigma r}\left[A^{*}\left(\lambda_{j}\right) \Delta \lambda_{j} L_{\lambda, p h, \text { illum }}\left(\lambda_{j}\right)\right]}{\max _{j}\left[A^{*}\left(\lambda_{j}\right) \Delta \lambda_{j} L_{\lambda, \text { ph, illum }}\left(\lambda_{j}\right)\right]} .
$$

This definition of dynamic range is illustrated in Figure 2 b) and c) for illuminant spectra representing daylight and halogen light. The quantity (8) could be termed "effective dynamic range" for the given illuminant, for example "effective dynamic range for solar illumination" which could be denoted $D R_{s u n}$. The dynamic range defined in (8) represents the allowable range of variation for the product of illumination level and reflectance. The definition (8) is thus a relevant characteristic for the dynamic range achievable in an application, at the expense of having to assume a particular illumination spectrum. To represent dynamic range for different illuminants, multiple values will have to be specified.
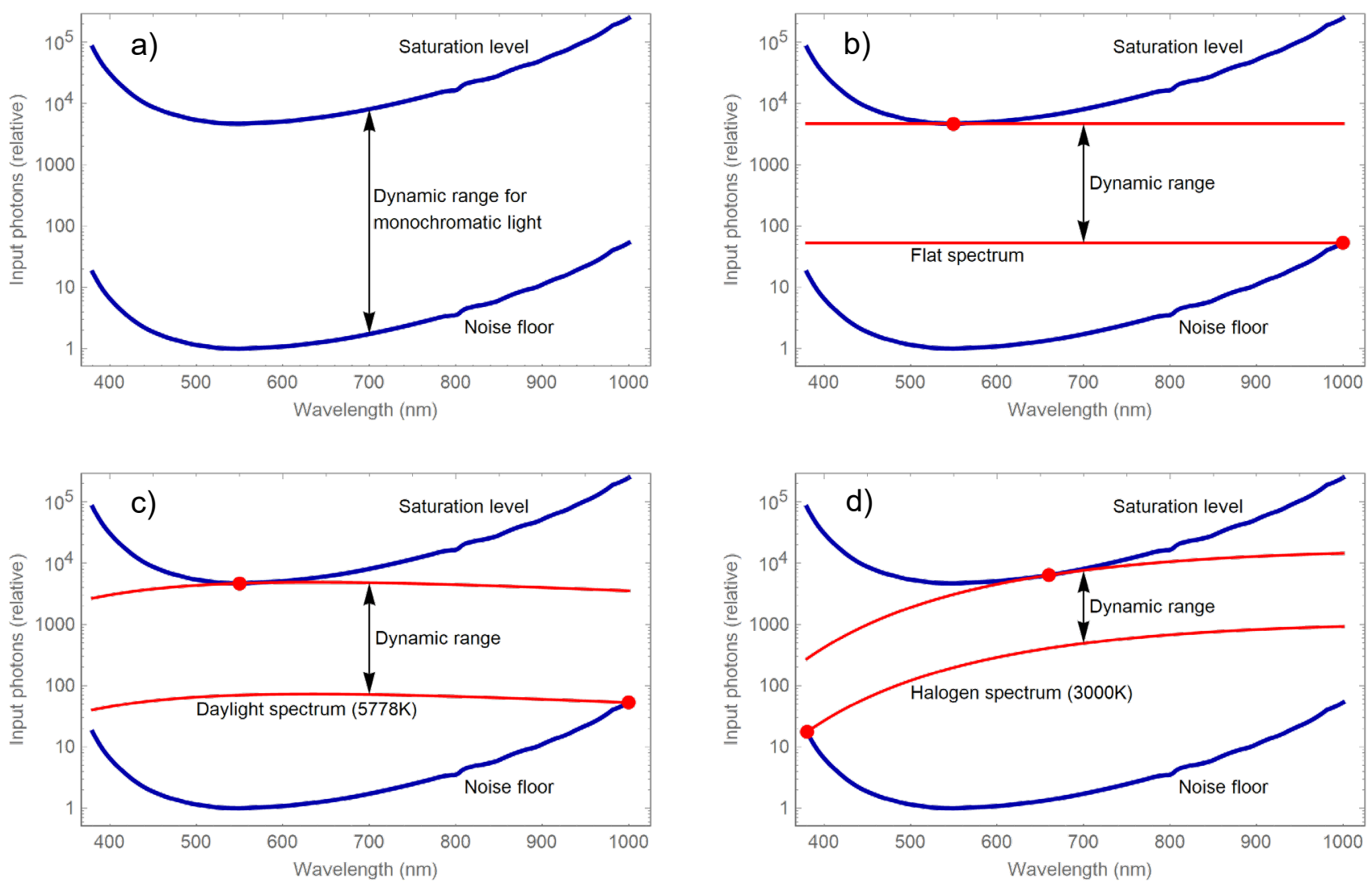

Figure 2. Graphs of saturation level and noise floor (blue) for an example system with the Effective throughput shown in Figure 1, plotted with incoming spectra (red) of different shapes and strengths. a) Monochromatic light at any single wavelength. b) Equal photon radiance across the spectral range. c) Daylight spectrum. d) Halogen spectrum. Red dots indicate points where an incoming spectrum reaches the noise floor or saturation level.

\section{DISCUSSION}

It is common in applications to have a situation where both light level and camera effective throughput drops off at one or both ends of the spectral range, such as in the cases shown in Figure 2 b) and c). It is well known that this spectral behavior tends to reduce the effective dynamic range. Of course, the full dynamic range can be recovered if the wavelength dependence of the illumination is opposite that of the effective throughput, but this is normally not an option. 
A common remedy is instead to employ equalization filters to attenuate the light in spectral regions where the product of light level and effective throughput is highest. Dynamic range can thus be improved at the expense of some loss of light. In the context of camera specification, it would then be possible to give two sets of values for dynamic range and effective throughput, with and without an equalizing filter.

Apart from spectral equalization filters, there are a number of ways to achieve "high dynamic range" (HDR) through extra functionality on the image sensor, such as multiple exposures or multiple sensitivities in each detector element. These techniques essentially work by increasing the saturation level. In spectral imaging, it is particularly attractive to be able to increase the saturation level only for detector elements that receive light in bands that tend to have the strongest photoelectron signal. Some systems have such an option. For example, some space systems can set gain for individual rows of detectors. The dynamic range as defined in (8) is still a relevant characteristic for the overall dynamic range of such HDR systems.

The dynamic range (8) can be considered optimistic since it does not include geometrical variation of throughput across the field of view due to geometric effects such as $\left(\cos ^{4}\right)$ dependence and vignetting. This could be included in the definition of dynamic range, but the definition would then involve a distinctly separate aspect of camera performance. In the context of camera specification, it is therefore preferable to describe dynamic range and geometrical throughput variation by separate characteristics. Then (8) still gives a meaningful value for dynamic range within a region of the field of view, assuming that the geometrical variation is smooth. The reduced dynamic range across the full field of view can be estimated with the aid of a characteristic for the geometrical variation.

To express dynamic range in the context of the P4001 standard, it is possible to use the definition in (7) which only makes reference to the camera properties. However, this definition essentially implies a flat photon radiance illumination spectrum, which is not representative of common application cases. Instead, or in addition, it is possible to define a set of application-relevant illumination sources to be used in the evaluation of dynamic range using (8). This will include spectra that approximate daylight and halogen lamp light, such as those in Figure 2, and could also include a flat energy radiance spectrum as a generalization of CIE illuminant $\mathrm{E}$.

Obviously, a specification of dynamic range must be verifiable by measurement. For this, the "photon transfer" technique $^{3}$ is directly applicable. Based on observed noise statistics, this technique can determine the noise floor and saturation level.

Finally, it can be noted that the definition of dynamic range according to (7) or (8) is derived from other camera characteristics that will anyway have to be specified. It is therefore not a major problem if cases occur where the definition of dynamic range is not applicable, for example if the readout noise is strongly dependent on operating conditions.

\section{CONCLUSION}

For a hyperspectral camera, a wide dynamic range is highly desirable to ensure good data quality. The dynamic range is therefore of interest as an element in the specification of camera performance, which the IEEE P4001 group is working to standardize. This paper has proposed a definition of dynamic range for a hyperspectral camera that takes into account the wavelength dependence of the effective throughput of the camera, as well as a definition that also considers the illuminant spectrum for a particular application. These characteristics for dynamic range are potentially interesting to hyperspectral camera users, for example for comparing the application performance of two hyperspectral cameras.

\section{ACKNOWLEDGMENTS}

Thanks are due to Harald Hovland of FFI and Emmett Ientilucci of RIT for constructive comments and discussions. 


\section{REFERENCES}

[1] IEEE Standards Association, "Standard for Characterization and Calibration of Ultraviolet through Shortwave Infrared (250 $\mathrm{nm}$ to $2500 \mathrm{~nm})$ Hyperspectral Imaging Devices," https://sagroups.ieee.org/sagroupshyperspectral

[2] Skauli, T., "Specifying radiometric performance of hyperspectral and conventional cameras: a minimal set of independent characteristics," Proc. SPIE 11392, Algorithms, Technologies, and Applications for Multispectral and Hyperspectral Imagery XXVI, 113920B (2020).

[3] Janesick, J. R., [Photon Transfer], SPIE Press (2007) 